\title{
Articulating Factors Defining RMS Delay Spread in LV PLC Networks
}

\author{
Sabih Güzelgöz, Hasan Basri Çelebi, and Hüseyin Arslan \\ Department of Electrical Engineering, University of South Florida, 4202 East Fowler Avenue, ENB-118, Tampa, FL 33620, USA \\ Correspondence should be addressed to Sabih Güzelgöz, sguzelgo@mail.usf.edu
}

Received 26 April 2010; Accepted 10 August 2010

Academic Editor: Abdallah Shami

Copyright (C) 2010 Sabih Güzelgöz et al. This is an open access article distributed under the Creative Commons Attribution License, which permits unrestricted use, distribution, and reproduction in any medium, provided the original work is properly cited.

\begin{abstract}
Communication over the power line network (PLN) referred as power line communication (PLC) has a long history of narrowband applications. With the recent developments in the field of digital communications, current interest is to exploit this medium for wideband communications for several applications such as Internet access, home networking, and in-vehicle data communication. In line with this recently emerging interest which envisions the conversion of a power transmission network into a communication network, understanding the root-mean-squared (RMS) delay spread is essential for multipath PLC channels for the establishment of reliable communication systems. In this paper, factors that play a role on the RMS delay spread value of low voltage (LV) PLC channels are articulated. Among these factors, dependency of the RMS delay spread on attenuation, loading, and physical characteristics of the communication channel in the PLNs is investigated.
\end{abstract}

\section{Introduction}

Communication over the power line network (PLN) referred as power line communication (PLC) is recently gaining significant momentum for various communication applications such as Internet, data and voice transmission [1]. PLC is very promising for many communication applications in the sense that the communication medium is based on the use of an existing infrastructure in a very extensive network that virtually reaches anywhere in the world. Design purpose of the PLNs is the transmission of power from one point to another, and conveying the high frequency communication signals is not the actual reason behind its existence. Therefore, PLN is considered to be a very harsh communication medium such that PLC systems must tackle various challenges such as noise and multipath in order to ensure reliable communication.

Future PLC-based systems are envisioned to provide very high data rates requiring wideband to support high-quality multimedia. The popularity of wideband PLC especially in low voltage (LV) networks for last-inch applications is growing $[2,3]$. In wideband communication channels, multipath induced intersymbol interference (ISI) is one of the phenomena that leads to performance degradation. In communications community, significance of ISI is quantified by a parameter called root-mean-squared (RMS) delay spread. In a nutshell, the RMS delay spread indicates the capability of the communication channel of supporting high data rate communications by implying the probability of performance degradation which may occur due to the ISI as a result of multipath signal propagation. Understanding the RMS delay spread in PLC channels is crucial from many aspects. For instance, the driving technology in PLC applications requiring wideband is overwhelmingly considered to be orthogonal frequency division multiplexing (OFDM) due to its inherent advantages [1]. Cyclic prefix is employed in OFDM systems in order to combat multipath related ISI, and RMS delay spread of the communication channel is considered while determining the duration of the cyclic prefix [4]. The apparent relation between RMS delay spread and the cyclic prefix in OFDM systems is one of the considerations that favors the idea of looking into RMS delay spread of LV PLC channels in a more detailed way. In PLC systems, the RMS delay spread depends on the following factors: 
(1) frequency-distance-dependent cable attenuation,

(2) loading condition, that is the impedances of the electrical loads appearing at termination points,

(3) physical characteristics of the operating PLC medium.

The aim of this study is to investigate and explain statistically the impact of these factors on the RMS delay spread value of LV PLC networks.

Based on extensive measurements, frequency-distance dependent attenuation in LV PLC networks is defined as [5]

$$
A(f, d)=\exp \left(\left(-a_{0}-a_{1} f^{k}\right) d\right),
$$

where $f$ and $d$ correspond to frequency of the signal and the distance covered, respectively. $a_{0}, a_{1}$, and $k$ are all cabledependent parameters and are mostly extracted by empirical measurements [5]. $a_{0}, a_{1}$, and $k$ are considered to be time invariant, that is, fixed parameters for a PLC network. Hence, attenuation defined by (1) does not cause any time variation in the RMS delay spread for a given topology. Unlike the first factor listed above, loading in LV PLC networks is time varying. Branches in the network are terminated with various electrical loads with different impedance characteristics. The loading condition of the network may change throughout the day. Hence, RMS delay spread of the LV PLC networks may change significantly as the impedances seen at the termination points change even if the considered network topology is fixed. In addition, even if the loading condition is not altered, the dependency of the load impedances on the Alternating Current $(\mathrm{AC})$ mains cycle [6] gives rise to a cyclic change in the RMS delay spread of PLC channels. Indeed, several studies performed earlier noticed this relation between RMS delay spread and loading by considering some specific topologies [7, 8]. An excerpt taken from [8] says:

\section{"It has been shown the maximum delay spread occurs for cases when the channel is terminated either in low impedances or high impedances".}

Some other studies available in the literature implicitly express this fact by calling the open circuit condition at termination points as the worst case scenario [9]. Findings of this study will naturally shed some light on these statements extensively encountered in the PLC community through the establishment of a mathematical background while the impact of loading is examined. In addition, PLC systems are deployed on PLNs possessing different physical characteristics. Number of branching nodes between transmitter and receiver, length statistics of branches, and statistics regarding the number of branches extending from each branching node are some of the characteristics which may significantly differ from network to network. Depending upon the communication application and deployment condition, distance between transmitter and receiver is another parameter which may also change. These different attributes of the PLNs give rise to a change in the multipath profile of the communication channel between transmitter and receiver leading to a change in the RMS delay spread values.
Revealing the relation between these physical characteristics of PLNs and the RMS delay spread is another objective of this study.

The remainder of the paper is organized as follows. Section 2 gives the analytical background for the multipath propagation model in PLC channels and defines RMS delay spread. Section 3 discuses impact of attenuation and loading on the RMS delay spread in PLC channels. Section 4 provides the details on the relation between the physical characteristics of PLC channels and RMS delay spread. Finally, the concluding remarks are given in Section 5.

\section{PLC Multipath Channel Model and RMS Delay Spread}

When a signal is transmitted on the power line conductors, the signal received at the receiver consists of attenuated, delayed, and phase-shifted replicas of the transmitted signal. If the total number of received signal replicas are considered to be limited to $N$, a complete characterization of the PLC channel can be given by its channel frequency response (CFR) as follows [5]:

$$
H(f)=\sum_{i=0}^{N}\left[\prod_{k=1}^{K} \Gamma_{i k} \prod_{m=1}^{M} T_{i m}\right] A\left(f, d_{i}\right) \exp \left(-j 2 \pi f \tau_{i}\right)
$$

where $\Gamma$ and $T$ correspond to the reflection and transmission coefficients along the propagation path, respectively, $A\left(f, d_{i}\right)$ means the frequency-distance dependent attenuation stemming from the physical characteristics of the cable used in the network and given by $(1)$, and $\exp \left(-j 2 \pi f \tau_{i}\right)$ refers to the phase of the $i$ th component due to the time delay. Finally, $K$ and $M$ represent the number of reflection and transmission coefficients experienced by a propagating signal along a particular path denoted by the subscript $i$. Multiplication of reflection $(\Gamma)$ and transmission $(T)$ coefficients leads to a parameter which is called reflection factor in the literature. Reflection factor, denoted as $\left|r_{i}\right| \exp \left(j \theta_{i}\right)$ for a certain path implied by the subscript $i$, is usually but not necessarily a complex number. It accounts for the losses inflicted upon the transmit signal due to physical characteristics of the PLC environment. It is clear from (2) that the accurate computation of the reflection factors (reflection and transmission coefficients) is essential for a true characterization of the PLC channel. In PLC systems, a transmit signal propagating from one location to another suffers reflections at impedance discontinuities along its path to the receiver. Due to these impedance mismatches, some part of the signal is reflected back towards the source whereas some proceeds to the destination. Reflection coefficient $\Gamma$ articulates the amplitude/phase ratio between the reflected signal and the incident signal whereas transmission coefficient $T$ implies the amplitude/phase relation between the proceeding signal and the incident signal. Reflection and transmission coefficients at a particular impedance discontinuity for a cable 

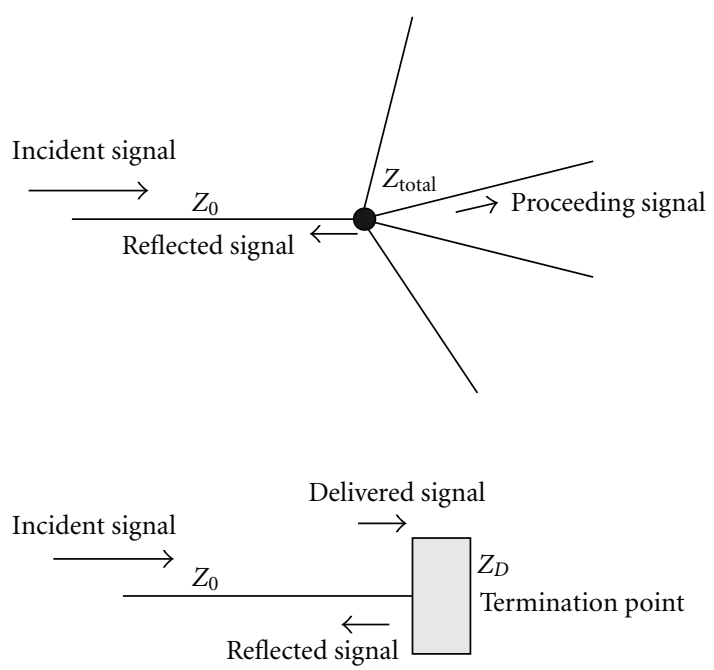

FIGURE 1: Reflection/transmission coefficients at branching and termination.

with characteristic impedance $Z_{0}$ are given by the following equations [10]:

$$
\Gamma=\frac{Z_{L}-Z_{0}}{Z_{L}+Z_{0}}, \quad T=1+\Gamma,
$$

where $Z_{L}$ refers to the impedance that the signal sees at a discontinuity. Since branching and impedance appearing at the termination points are the main reason behind the impedance discontinuity for homogeneous PLNs which are to be considered in our study, it is worth taking a closer look at the calculation of the reflection and transmission coefficients at these instants as illustrated in Figure 1.

2.1. Reflection/Transmission Coefficient at Branching. At a branching node, calculation of reflection/transmission coefficient is based on treating each branch extending from the node as parallel connection. If a homogeneous network with a particular cable impedance $Z_{0}$ is assumed, it is easy to show that the impedance seen by the incident signal arriving at a branching node is given by the following expression:

$$
Z_{\text {total }}=\frac{Z_{0}}{n-1}
$$

where $n$ is the total number of branches extending from a node including the branch on which the incident signal propagates (Note that 3 is the minimum value that $n$ can assume to ensure branching keeping in mind that a node should have at least one branch for arriving signal and one branch for proceeding signal.). By using (3), it can be easily shown that reflection and transmission coefficients are given by

$$
\Gamma=\frac{Z_{\text {total }}-Z_{0}}{Z_{\text {total }}+Z_{0}}=\frac{2-n}{n}, \quad T=\Gamma+1=\frac{2}{n},
$$

2.2. Reflection/Transmission Coefficient at Termination Points. In a similar fashion, reflection/transmission coefficient at a termination node can be computed as follows:

$$
\Gamma=\frac{Z_{D}-Z_{0}}{Z_{D}+Z_{0}}, \quad T=\Gamma+1=\frac{2 Z_{D}}{Z_{D}+Z_{0}},
$$

where $Z_{D}$ denotes the impedance seen by the incident signal at the termination point. Note that the incident wave is fully reflected in case the termination point is open or short with the same amplitude but $180^{\circ}$ of phase difference. In case a device is connected to the termination point, then impedance of the corresponding device must be taken into consideration while computing reflection/transmission coefficients. Signal passes through many impedance discontinues and experiences multiple reflections on its way to the receiver in a PLC network. Reflection factor, that was previously mentioned while introducing the multipath characteristics of PLC channels, represents the total effect of all these reflection/transmission coefficients on the propagating signal.

If inverse fast Fourier transform (IFFT) operation is applied on CFR given by (2), channel impulse response (CIR) is obtained as follows:

$$
h(\tau)=\sum_{j=0}^{R} h_{j} \delta\left(t-\tau_{j}\right) .
$$

RMS delay spread is derived from the CIR and defined as [11]

$$
\tau_{\mathrm{rms}}=\sqrt{\frac{\sum_{j=0}^{R} \tau_{j}^{2}\left|h_{j}\right|^{2}}{\sum_{j=0}^{R}\left|h_{j}\right|^{2}}-\left(\frac{\sum_{j=0}^{R} \tau_{j}\left|h_{j}\right|^{2}}{\sum_{j=0}^{R}\left|h_{j}\right|^{2}}\right)^{2}},
$$

where $R$ is the number of paths considered in the calculation and usually determined by thresholding as will be clear in the forthcoming sections. It is worth mentioning that delay of the first arriving path, which is denoted as $\tau_{0}$, is aligned to zero before computation.

So far, signal propagation and multipath characteristics of PLC channels as well as the computation of the RMS delay spread are elaborated. These concepts are important in a sense that they form a basis for our future discussions. From this point on, our discussion will be extended to the RMS delay spread and the factors that characterize it in PLNs.

\section{Impact of Attenuation and Loading on RMS Delay Spread}

Our initial objective is to understand the impact of attenuation and loading on the RMS delay spread. T-network is the main PLC topology in LV PLC networks that is usually taken into consideration. Multipath phenomenon was first elaborated by considering a T-network topology in [5]. Similarly, signal reflections are analyzed by considering this fundamental topology structure in [12]. Its popularity in PLC channel analysis stems from the fact that it forms a basis for more complex LV networks in a way that these complex networks can be decomposed into different connections of T-network structure $[5,12]$. Stemming from the fact that 


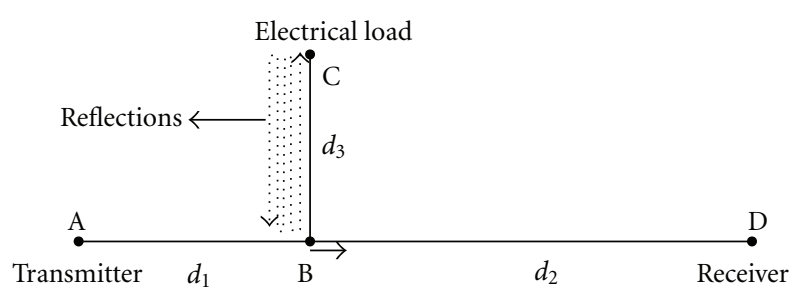

FIgURE 2: T-network topology.

our initial objective is to investigate the impact of attenuation and loading on the RMS delay spread, physical characteristics of PLC channels will be isolated from our analysis and popularly used T-network topology, being a more controlled environment, will be utilized in line with the previous studies.

T-network topology is depicted in Figure 2. It is composed of three branches connected to node $B$ with the length of $d_{1}, d_{2}$, and $d_{3}$. A denotes the point where the signal is injected into the network, and $D$ is the point where the signal is received. Consideration of the homogeneous network structure in which all the branches have the same characteristic impedance $Z_{0}$ is one of the assumptions made for the ease of analysis. In addition, $A$ and $D$ are assumed to be matched to $Z_{0}$ for the sake of simplicity, hence $B$ and $C$ are the only sources of reflection in the topology.

Reflection and transmission coefficients at node $B\left(\Gamma_{b}\right.$, $\left.T_{b}\right)$ and $C\left(\Gamma_{c}, T_{c}\right)$ for this particular network topology are given by (5) and (6) for $n=3$. Note that $Z_{D}$ in (6) refers to impedance of the electrical load connected to node $C$.

Following the discussion on PLC channel and RMS delay spread given in Section 2, it is now convenient to articulate why the first two factors (attenuation and loading) that are listed in Section 1 play a role in the RMS delay spread of PLC channels. Recall that the RMS delay spread of a communication channel is computed by aligning the first arriving path to zero delay. Upon this alignment, the number of paths to be included in the RMS delay spread computation, which is $R$ as can be seen in (8), is determined by applying a threshold considering the maximum power value in the delay profile. With this threshold so determined, the paths with the power values below are considered to be noise and excluded from the analysis. With the explanation given, the impact of attenuation on RMS delay spread can be explained by an example. Considering the T-network topology given in Figure 2, assume that only two paths satisfy the threshold condition. So, these paths should naturally follow the following paths: A-B-D (at $\tau_{0}$ ) and A-B-C-B-D (at $\left.\tau_{1}\right)$. Assume that $d_{3}$ is slowly increased. Note that this change in the topology does not lead to any change in either $\tau_{0}$ or in the power of the first arriving path since $d_{1}+d_{2}$ stays the same. However, this affects the second arriving path in a way that it arrives at a later delay with a smaller value of power due to the attenuation which increases with distance. The attenuation may reach at a point $\left(\right.$ as $\left.d_{3} \rightarrow \infty\right)$ at which the threshold condition is not satisfied even for the second arriving path leading us to consider it as a part of background noise in the computation of RMS delay spread.
Disappearance of a particular path from delay profile (or arriving at a larger delay) leads to a change in the RMS delay spread value as can be clearly seen from (8).

The second factor given in Section 1, which is the loading, determines $\Gamma$ 's and T's in (2). Therefore, any change in the loading condition leads to a change in $\Gamma$ 's and T's in the network and results in a change in the RMS delay spread. Recall also that even if the loading condition is not altered, the dependency of the load impedances on the AC mains cycle [6] gives rise to a cyclic change in the RMS delay spread of PLC channels. If the term $A(f, d)$ in (2) is ignored in order to isolate ourselves from the effect of attenuation and solely focus on the impact of loading, (2) reduces to the following form:

$$
H(f)=\sum_{i=0}^{N}\left[\prod_{k=1}^{K} \Gamma_{i k} \prod_{m=1}^{M} T_{i m}\right] \exp \left(-j 2 \pi f \tau_{i}\right) .
$$

If IFFT operation is applied to the CFR, CIR is obtained as follows:

$$
h(\tau)=\sum_{i=0}^{N}\left[\prod_{k=1}^{K} \Gamma_{i k} \prod_{m=1}^{M} T_{i m}\right] \delta\left(t-\tau_{i}\right)
$$

Note that the reflection factor of the direct path (A-B-D) is composed of only one term which is the transmission coefficient at $B$, namely, $T_{b}$. The reflection factors of other paths consist of $\Gamma_{c}, \Gamma_{b}$, and $T_{b}$. Based upon this observation, the CIR of T-network topology assumes the following form:

$h(\tau)=T_{b} \delta\left(\tau-\tau_{0}\right)+\sum_{j=1}^{R} T_{b}\left\{\frac{2}{n}\right\}^{j}\left\{\frac{2-n}{n}\right\}^{j-1}\left(\Gamma_{c}\right)^{j} \delta\left(\tau-\tau_{j}\right)$,

where $R$ refers to the number of reflections considered between $B$ and $C$.

The graphical illustration of the amplitude of (11) is given in Figure 3. As can be seen, amplitude of the signal components decreases with increasing delay. This is due to the fact that power of the signal reduces as it passes through more reflection points on its way to the receiver even though the attenuation effect is not accounted for. Taking the attenuation into consideration, amplitude of each component should be even smaller. Therefore, the RMS delay spread values obtained from Figure 3 are pessimistic since the effect of attenuation especially for late arriving paths is not accounted for. However, this will not affect our conclusions since our focus is on the impact of loading rather than attenuation.

For the purpose of mathematical tractability, if two of the delays $\left(\tau_{0}\right.$ and $\left.\tau_{1}\right)$ along the delay axis are considered $(R=1)$ in Figure 3, using (8) and (11) the RMS delay spread $\tau_{\mathrm{rms}}$ takes the following form:

$$
\tau_{\mathrm{rms}}=\tau_{1} \frac{2 n\left|\Gamma_{c}\right|}{n^{2}+4\left(\Gamma_{c}\right)^{2}}=\tau_{1} \frac{6\left|\Gamma_{c}\right|}{9+4\left(\Gamma_{c}\right)^{2}} .
$$




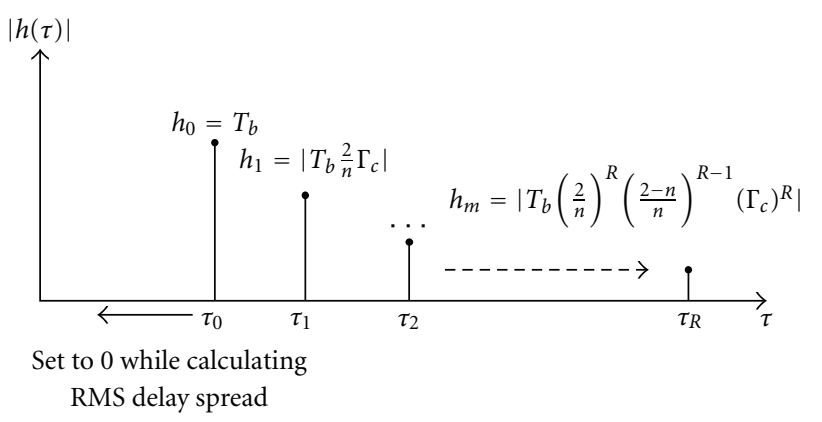

FIgURE 3: Graphical representation of the CIR for T-network topology.

Note that (12) depends on two parameters which are $\tau_{1}$ and the reflection coefficient $\Gamma_{c}$. When $\tau_{0}$ is set to 0 , time delay $\tau_{1}$ for the second arriving path is expressed as

$$
\tau_{1}=\frac{2 d_{3}}{v}=\frac{2 d_{3} \sqrt{\epsilon_{r}}}{c_{0}}
$$

where $\epsilon_{r}$ is the dielectric constant of the insulation material and $c_{0}$ is the speed of light in vacuum.

Referring back to (3), $\Gamma_{c}$ being the reflection coefficient at node $C$ depends on the impedance of the electrical device $Z_{D}$ connected to the network which is regarded as a random variable (RV) in this study for generalization so that various loading conditions can be taken into account. If $C$ is assumed to be left open $\left(Z_{D}=\infty\right), \Gamma_{c}$ becomes $1 . \Gamma_{c}$ becomes -1 if a short circuit assumption $\left(Z_{D}=0\right)$ is considered at node $C$. These two scenarios correspond to two extreme cases. Therefore, it is expected that any electrical device connected to node $C$ yields a reflection coefficient between the values generated by these extreme cases, -1 and 1 . These two values are also the maximum and minimum values of $\Gamma_{c}$ during an AC cycle duration even if the electrical load connected to node $C$ is unchanged.

In order to understand the impact of loading on the RMS delay spread $\tau_{\text {rms }}$, we have to derive the probability density function (PDF) of the variable

$$
\psi=\frac{6\left|\Gamma_{c}\right|}{9+4\left(\Gamma_{c}\right)^{2}} .
$$

Before proceeding with the statistical characterization of $\psi$, some important observations should be made regarding its behavior.

Lemma 1. $\psi$ has the following characteristics:

(i) monotonically increasing for $0<\Gamma_{c} \leq 1$,

(ii) monotonically decreasing for $-1 \leq \Gamma_{c}<0$,

(iii) has a critical point at $\Gamma_{c}=0$.

Proof. $\psi$ takes the following form within this interval $0<$ $\Gamma_{c} \leq 1:$

$$
\psi=\frac{6 \Gamma_{c}}{9+4\left(\Gamma_{c}\right)^{2}}
$$

The derivative of $\psi$ with respect to $\Gamma_{c}$ is given by

$$
\frac{d \psi}{d \Gamma_{c}}=\frac{54-24\left(\Gamma_{c}\right)^{2}}{\left(9+4\left(\Gamma_{c}\right)^{2}\right)^{2}},
$$

Since $d \psi / d \Gamma_{c}>0$ for $0<\Gamma_{c} \leq 1, \psi$ is a monotonically increasing function within this interval.

Similarly, the derivative of $\psi$ with respect to $\Gamma_{c}$ within the interval $-1 \leq \Gamma_{c}<0$ is given by

$$
\frac{d \psi}{d \Gamma_{c}}=\frac{-54+24\left(\Gamma_{c}\right)^{2}}{\left(9+4\left(\Gamma_{c}\right)^{2}\right)^{2}} .
$$

Since $d \psi / d \Gamma_{c}<0$ for $-1 \leq \Gamma_{c}<0, \psi$ is a monotonically decreasing function within this interval.

Equating (16) and (17) shows that $\psi$ has a critical point at $\Gamma_{c}=0$, where the function may have a minimum or maximum. However, it is easily seen from the first and second claims that $\Gamma_{c}=0$ minimizes $\psi$. It is also worth mentioning that $\left|\Gamma_{c}\right|=1$ maximizes $\psi$ considering its behavior on the intervals analyzed.

An alternative insight into the lemma can be given in this way. $\Gamma_{c}=0$ corresponds to the case in which the impedance of the electrical device connected at node $C$ matches the impedance of the cable $Z_{0}$. This case implies that no reflection occurs at $C$ leaving only one propagation path between $A$ and $D$. Disappearance of the other signal components along the delay axis in Figure 3 gives rise to minimum value of the RMS delay spread, hence $\psi$. Other values of $\Gamma_{c} \neq 0$ lead to the reception of several signal components from other propagation paths along with the direct path. This phenomenon leads to an increase in the value of the RMS delay spread, hence $\psi$. First and the second claims are details of this observation. Claims derived from our analytical analysis also shed some light on the statements that are encountered frequently in the literature as mentioned in Section 1. As empirically concluded in the previous studies and mathematically shown here, open/short circuit condition at the termination point leads to the maximum value of RMS delay spread whereas its minimum value is obtained if the termination points are matched to the network cable impedance. This also validates the reason why open/short circuit condition at termination points of LV PLC channels is considered as the worst case scenario from the perspective of multipath signal propagation.

Referring back to the statistics of $\psi$, several PDFs can be offered in order to characterize the statistical behavior of $\Gamma_{c}$. Extracting the statistics of device impedances that are widely used in LV networks and building a statistical model would be very desirable. Since no such study is available in the literature, $\Gamma_{c}$ is assumed to be uniformly distributed over $[-1,1]$. By using the fundamental theorem for functions of one random variable [13] and employing change of variables 


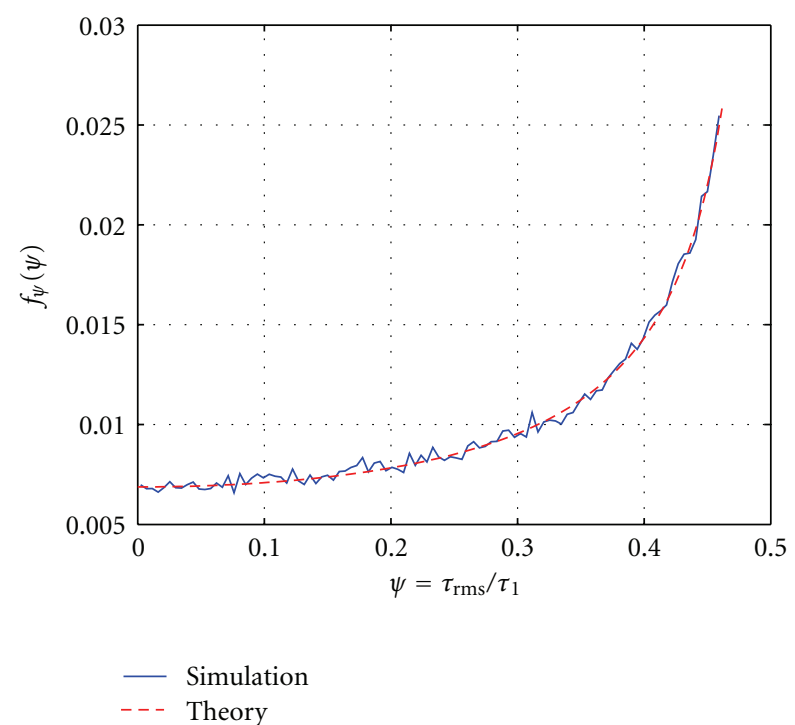

FIGURE 4: PDF of the RMS delay spread of T-network topology when node $C$ is randomly loaded.

$Y=\left|\Gamma_{c}\right|$, the PDF of (14) can be expressed as follows:

$$
f_{\psi}(\psi)=\frac{\left(36 \psi^{2}+\left(3-3 \sqrt{1-4 \psi^{2}}\right)^{2}\right)^{2}}{24 \psi^{2}\left(36 \psi^{2}-\left(3-3 \sqrt{1-4 \psi^{2}}\right)^{2}\right)}, \quad 0<\psi<\frac{6}{13} .
$$

Integrating (18) leads to the cumulative distribution function $(\mathrm{CDF})$ of $\psi$. CDF can be calculated by changing the variable $\cos (\theta)=\sqrt{1-4 \psi^{2}}$. After a series of cumbersome mathematical operations that are skipped here for the sake of brevity, $\mathrm{CDF}$ is computed as

$$
F_{\psi}(\psi)=\frac{3}{2} \tan \left(\frac{\arcsin (2 \psi)}{2}\right), \quad 0<\psi<\frac{6}{13} .
$$

Figures 4 and 5 show the PDF and CDF of $\psi$. It is clearly seen that analytical derivations and simulation results are in good agreement. Curves are also seen to be in good agreement with the lemma provided. For instance, plugging $\left|\Gamma_{c}\right|=1$ into (14) for the purpose of maximization yields $6 / 13$. Due to the maximization, $\psi$ must never exceed $6 / 13$ and it is seen from Figure 5 that this observation is satisfied.

Impact of attenuation and loading on the RMS delay spread is detailed in this section. Subsequently, impact of the physical characteristics of the PLC operating environment will be our focus.

\section{Impact of the Physical Characteristics of the PLC Channel on RMS Delay Spread}

PLC systems may be deployed on LV PLC environments having entirely different physical characteristics. Among these characteristics, impact of the following items on the RMS delay spread will be addressed in this section:

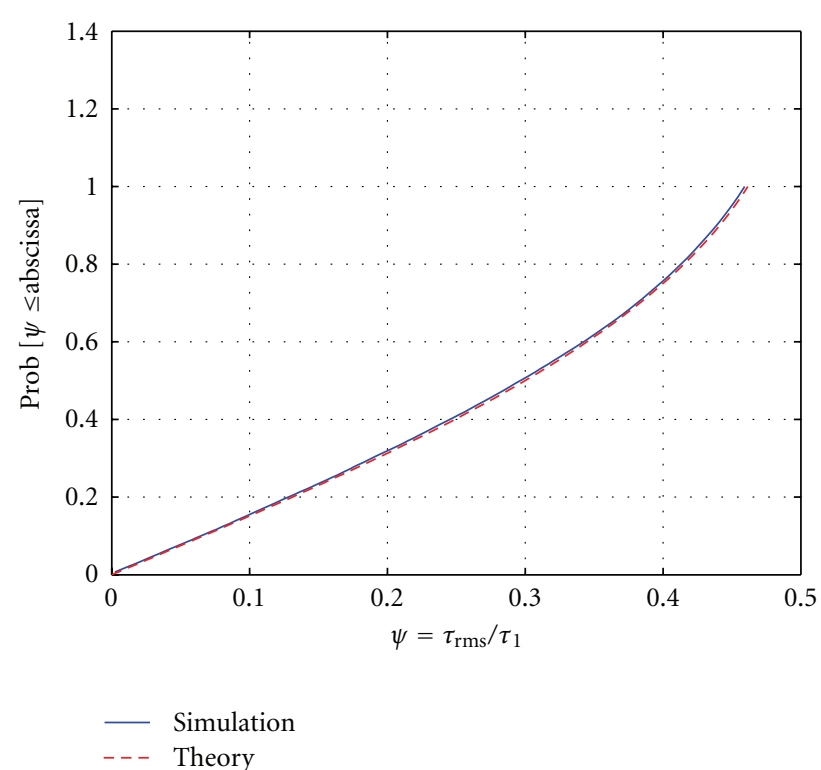

Figure 5: CDF of the RMS delay spread of T-network topology when node $C$ is randomly loaded.

(i) number of branching nodes between transmitter and receiver,

(ii) distance between transmitter and receiver,

(iii) length statistics of branches.

It is obvious that the analysis that we plan to perform in this section requires the establishment of more complicated PLC networks than the T-network topology utilized in Section 3. Modeling PLC systems and building simulation techniques for them have been the focus of several studies earlier in the literature. The model which considers the PLC channel as a multipath communication environment was first introduced in [5] as mentioned earlier. Based upon this multipath consideration, analytical calculation of the multipath components by describing the PLC channel via a set of matrices is proposed in $[14,15]$. PLC models that are based on treating the transmission line as a two-port device are available in the literature as well $[16,17]$. A channel model and a simulation platform along with the results of various channel measurement campaigns are discussed in $[18,19]$. A statistical PLC channel characterization regarding attenuation, multipath related parameters, and so forth, is presented in $[20,21]$. In our analysis, the matrix-based PLC simulation technique proposed in [14] will be considered as the basis. However, matrices introduced in [14] are to be modified in a way that the simulation module lets us easily generate PLC networks with different physical characteristics. In line with the procedure described in [14], generated PLC network topology, which is illustrated in Figure 6, is mapped into a single matrix as follows:

$$
\mathrm{C}_{[(\mathrm{t}+\mathrm{b}) \times(\mathrm{t}+\mathrm{b})]}=\left[\begin{array}{cc}
0_{[t \times t]} & C T_{[t \times b]} \\
C T_{[b \times t]}^{T} & C C_{[b \times b]}
\end{array}\right],
$$

where $t$ and $b$ correspond to the number of termination points and branching points (referred to as internal nodes 


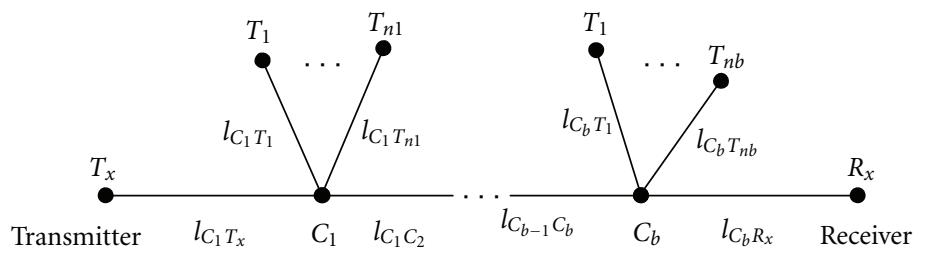

FIgURE 6: Graphical illustration of the PLC network topology considered in the study.

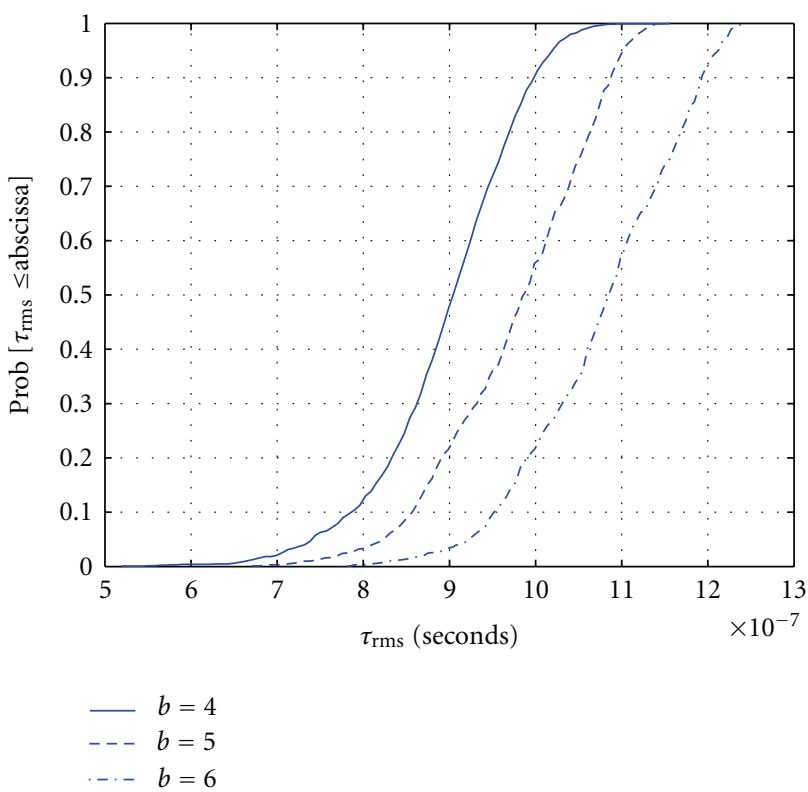

Figure 7: Dependency of RMS delay spread $\left(\tau_{\text {rms }}\right)$ on the number of nodes $(b)$ between transmitter and receiver. Separation distance between transmitter and receiver is $150 \mathrm{~m}$. Branch lengths are assumed to be uniformly distributed over [ $10 \mathrm{~m}-30 \mathrm{~m}]$.

in [14]), respectively. $C C$ is the submatrix which describes the interconnections among branching nodes. $C T$ shows the connections between branching nodes and termination points. The corresponding length of each interconnection and impedances at termination points are kept in separate matrices. In order to isolate our analysis from the impact of impedance variation that was discussed in Section 3 and focus solely on the impact of physical characteristics of the environment, it is assumed that the termination points are open circuit. In addition, number of branches extending from each branching node is considered to be uniformly distributed over $[3,6]$ in the simulations. Similar to the analysis performed in Section 3, transmitter and receiver are also assumed to be matched to the characteristic impedance of the homogeneous PLC network. Impact of physical attributes is statistically investigated by generating 20000 realizations of PLC network for each case taken into consideration. PLC topologies with different physical attributes are generated by manipulating the values of $t$, $b$, and the length matrix whose elements are composed of the values $l_{i j}$ shown in Figure 6. Note that a change

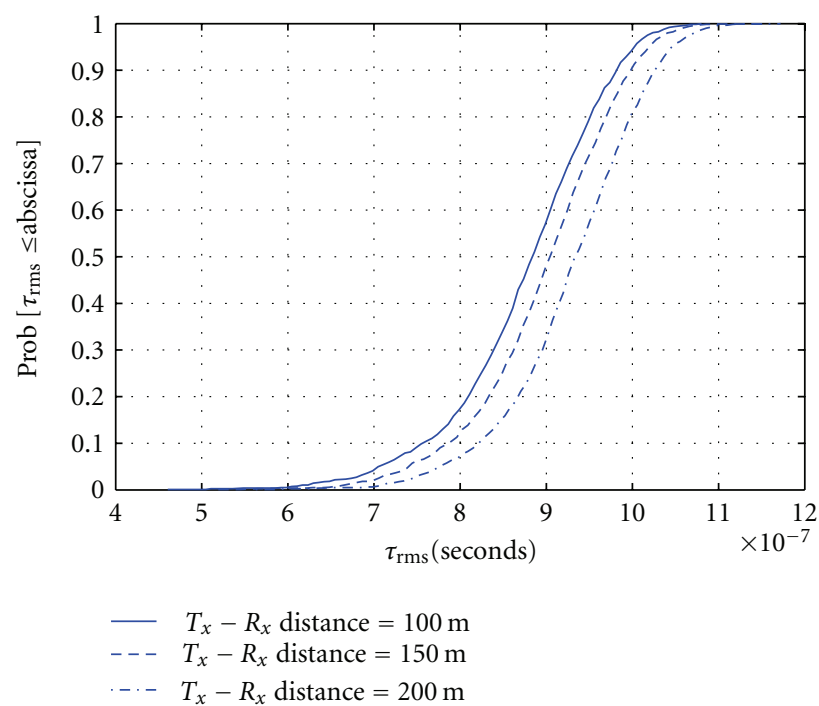

FIGURE 8: Dependency of RMS delay spread $\left(\tau_{\text {rms }}\right)$ on the separation distance $(d)$ between transmitter and receiver. Number of nodes between transmitter and receiver is 4 . Branch lengths are assumed to be uniformly distributed over [ $10 \mathrm{~m}-30 \mathrm{~m}]$.

in the topology gives rise to a change in the values of $t$ and $b$ which results in a change in the dimensions of the submatrices denoted as $C C$ and $C T$. For each realization, CIR was calculated by taking the IFFT of CFR given by (2). After CIR is obtained, procedure described in Section 2 is followed with the threshold value of $20 \mathrm{~dB}$ while computing the RMS delay spread.

The impact of number of nodes between transmitter and receiver on the RMS delay spread can be seen in Figure 7. While deriving this figure, transmitter-receiver separation distance and length statistics of the branches are considered to be $150 \mathrm{~m}$ and $\mathrm{U}[10 \mathrm{~m}-30 \mathrm{~m}$ ) (U refers to uniform distribution), respectively. Upon the analysis performed, it is concluded that an increase in the number of nodes while keeping all the other effective physical attributes of the PLC network the same gives rise to an increase in its RMS delay spread value. This behavior can be related to the multipath components arriving at larger delays as $b$ is increased. This relation was previously noticed in [7] by considering some specific PLC network topologies. Our findings verify the results of this earlier study by taking 


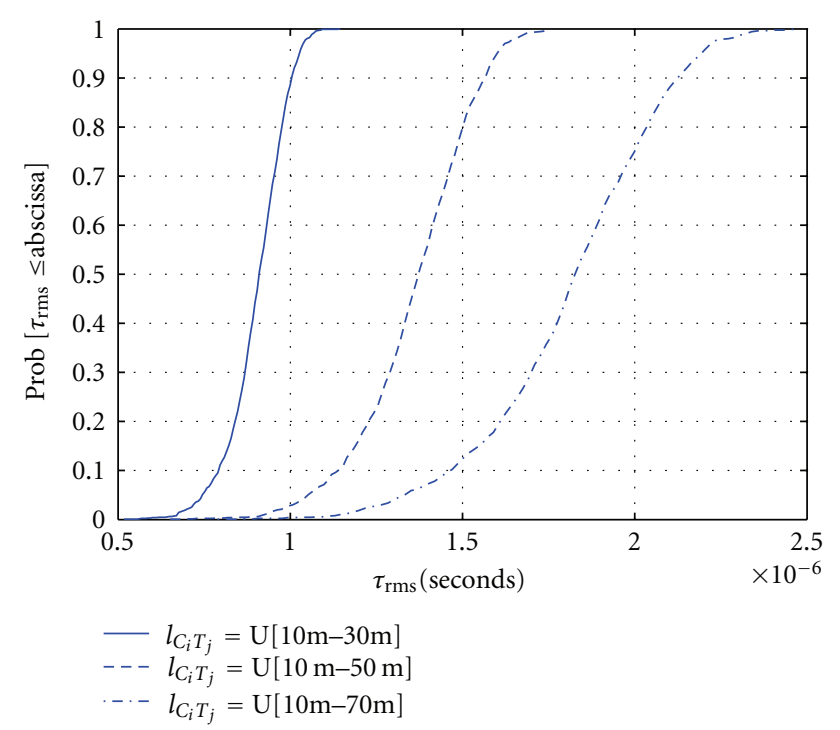

Figure 9: Dependency of RMS delay spread $\left(\tau_{\mathrm{rms}}\right)$ on the length statistics of branches. Number of nodes between transmitter and receiver is 4 . Separation distance between transmitter and receiver is $150 \mathrm{~m}$.

more general PLC network scenarios into account. Figure 8 shows the impact of transmitter-receiver separation distance on $\tau_{\text {rms }}$. Similar to the previous case analyzed, increasing separation distance between transmitter and receiver leads to the reception of multipath components at larger delays leading to an increase in $\tau_{\text {rms }}$ values. Finally, the impact of branch length statistics is seen in Figure 9. Among all of these aforementioned factors, change in the length statistics of branches seems to yield the most drastic change in $\tau_{\text {rms }}$ values. This is expected since expanding the range of values that branch lengths may assume increases the probability that some multipath components arrive at larger delays. If the effect of attenuation is not significant within the length range considered, it should naturally result in higher $\tau_{\mathrm{rms}}$ values as can be seen in the figure.

\section{Conclusion}

Trend in PLC indicates that the future applications will need more bandwidth and higher data rates to address user requirements. This growing interest into PLC entails a good understanding of its channel characteristics. RMS delay spread which is frequently used in communications community while quantifying multipath characteristics of communication channels was the focus of this study. Factors, which have a significant impact on the RMS delay spread, peculiar to PLC channels were elaborated in a detailed way. Among these factors, impact of attenuation and loading was discussed by exploiting T-network being the most fundamental LV PLC topology that is frequently used in the literature. The relation between attenuation and loading to the RMS delay spread was explicitly demonstrated and compared with the results already present in the literature.
Next, impact of the physical characteristics of PLC channels on the RMS delay spread was studied. Number of nodes between transmitter and receiver, transmitter-receiver separation distance, and length statistics of branches are identified as the physical characteristics which may significantly change from one PLC network to another. A matrix-based simulation technique was employed while generating PLC network topologies with distinct physical characteristics. For each attribute examined, statistics regarding the RMS delay spread were presented by observing corresponding CDF curves. Based upon the results of extensive simulations, important conclusions regarding the relation between these attributes and the RMS delay spread were drawn.

\section{References}

[1] N. Pavlidou, A. J. Han Vinck, J. Yazdani, and B. Honary, "Power line communications: state of the art and future trends," IEEE Communications Magazine, vol. 41, no. 4, pp. 34-40, 2003.

[2] S. Galli, A. Scaglione, and K. Dostert, "Broadband is power: internet access through the power line network," IEEE Communications Magazine, vol. 41, no. 5, pp. 82-83, 2003.

[3] Y.-J. Lin, H. A. Latchman, M. Lee, and S. Katar, "A power line communication network infrastructure for the smart home," IEEE Wireless Communications, vol. 9, no. 6, pp. 104-111, 2002.

[4] H. Schulze and C. Luders, Theory and Applications of OFDM and CDMA: Wideband Wireless Communications, Wiley, New York, NY, USA, 2005.

[5] M. Zimmermann and K. Dostert, "A multipath model for the powerline channel," IEEE Transactions on Communications, vol. 50, no. 4, pp. 553-559, 2002.

[6] F. J. C. Corripio, J. A. C. Arrabal, L. D. del Río, and J. T. E. Muñoz, "Analysis of the cyclic short-term variation of indoor power line channels," IEEE Journal on Selected Areas in Communications, vol. 24, no. 7, pp. 1327-1338, 2006.

[7] J. Anatory, N. Theethayi, and R. Thottappillil, "Channel characterization for indoor power-line networks," IEEE Transactions on Power Delivery, vol. 24, no. 4, pp. 1883-1888, 2009.

[8] J. Anatory, N. Theethayi, R. Thottappillil, and N. H. Mvungi, "A broadband power-line communication system design scheme for typical tanzanian low-voltage network," IEEE Transactions on Power Delivery, vol. 24, no. 3, pp. 1218-1224, 2009.

[9] I. C. Papaleonidopoulos, C. N. Capsalis, C. G. Karagiannopoulos, and N. J. Theodorou, "Statistical analysis and simulation of indoor single-phase low voltage power-line communication channels on the basis of multipath propagation," IEEE Transactions on Consumer Electronics, vol. 49, no. 1, pp. 89-99, 2003.

[10] D. M. Pozar, Microwave Engineering, John Wiley \& Sons, Toronto, Canada, 1998.

[11] T. S. Rappaport, Wireless Communications: Principles and Practice, Prentice-Hall, Upper Saddle River, NJ, USA, 1996.

[12] H. He, S. Cheng, Y. Zhang, and J. Nguimbis, "Analysis of reflection of signal transmitted in low-voltage powerline with complex wavelet," IEEE Transactions on Power Delivery, vol. 19, no. 1, pp. 86-91, 2004.

[13] A. Papoulis, Probability, Random Variables, and Stochastic Processes, McGraw-Hill, New York, NY, USA, 3rd edition, 1991. 
[14] D. Anastasiadou and T. Antonakopoulos, "Multipath characterization of indoor power-line networks," IEEE Transactions on Power Delivery, vol. 20, no. 1, pp. 90-99, 2005.

[15] X. Ding and J. Meng, "Channel estimation and simulation of an indoor power-line network via a recursive time-domain solution," IEEE Transactions on Power Delivery, vol. 24, no. 1, pp. 144-152, 2009.

[16] S. Barmada, A. Musolino, and M. Raugi, "Innovative model for time-varying power line communication channel response evaluation," IEEE Journal on Selected Areas in Communications, vol. 24, no. 7, pp. 1317-1325, 2006.

[17] H. Meng, S. Chen, Y. L. Guan et al., "Modeling of transfer characteristics for the broadband power line communication channel," IEEE Transactions on Power Delivery, vol. 19, no. 3, pp. 1057-1064, 2004.

[18] L. T. Tang, P. L. So, E. Gunawan, Y. L. Guan, S. Chen, and T. T. Lie, "Characterization and modeling of in-building power lines for high-speed data transmission," IEEE Transactions on Power Delivery, vol. 18, no. 1, pp. 69-77, 2003.

[19] F. J. Cañete, L. Díez, J. A. Cortés, and J. T. Entrambasaguas, "Broadband modelling of indoor power-line channels," IEEE Transactions on Consumer Electronics, vol. 48, no. 1, pp. 175$183,2002$.

[20] M. Tlich, A. Zeddam, F. Moulin, and F. Gauthier, "Indoor power-line communications channel characterization up to $100 \mathrm{MHz}$ - part I: one-parameter deterministic model," IEEE Transactions on Power Delivery, vol. 23, no. 3, pp. 1392-1401, 2008.

[21] M. Tlich, A. Zeddam, F. Moulin, and F. Gauthier, "Indoor power-line communications channel characterization up to $100 \mathrm{MHz}$ - part II: time-frequency analysis," IEEE Transactions on Power Delivery, vol. 23, no. 3, pp. 1402-1409, 2008. 

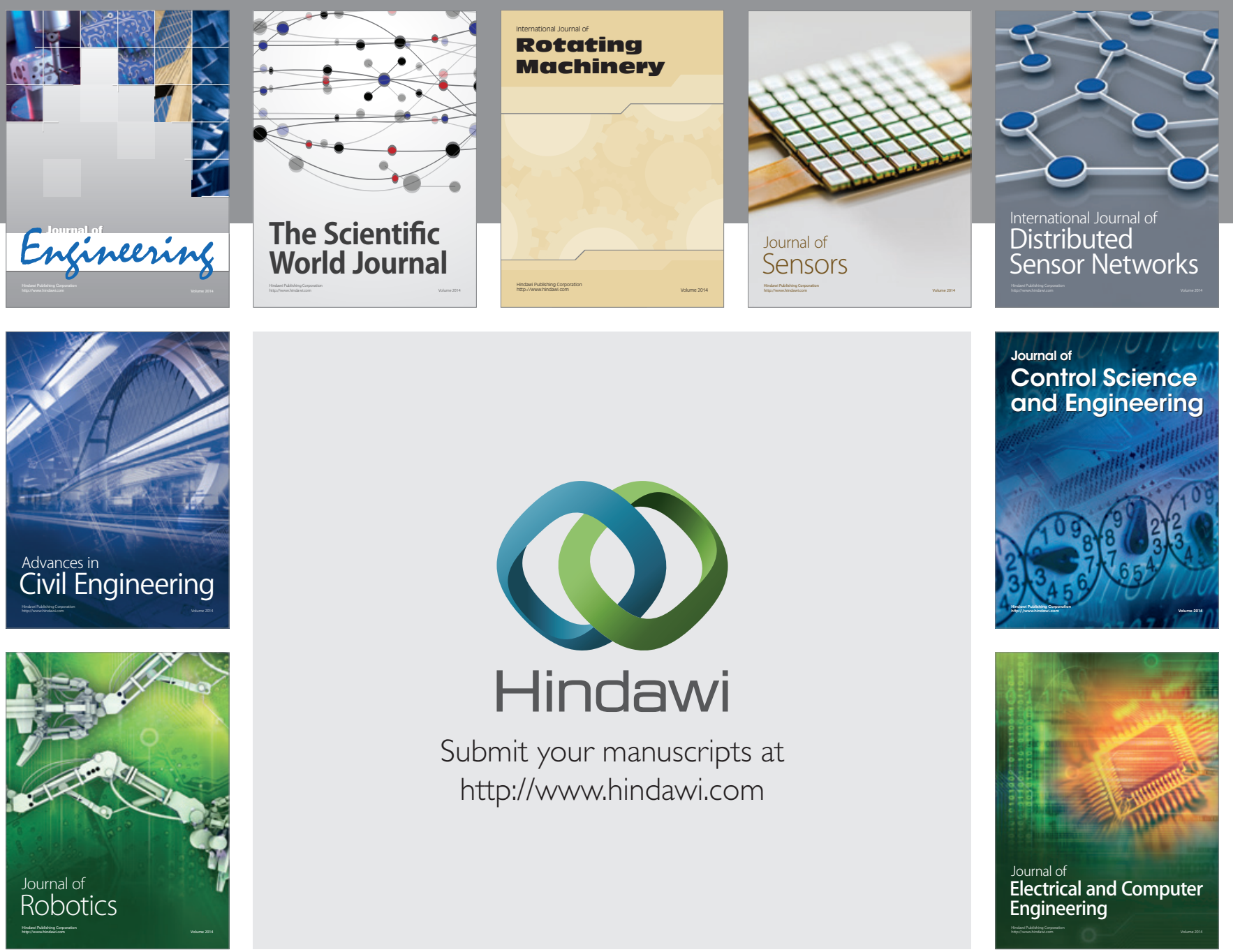

Submit your manuscripts at

http://www.hindawi.com
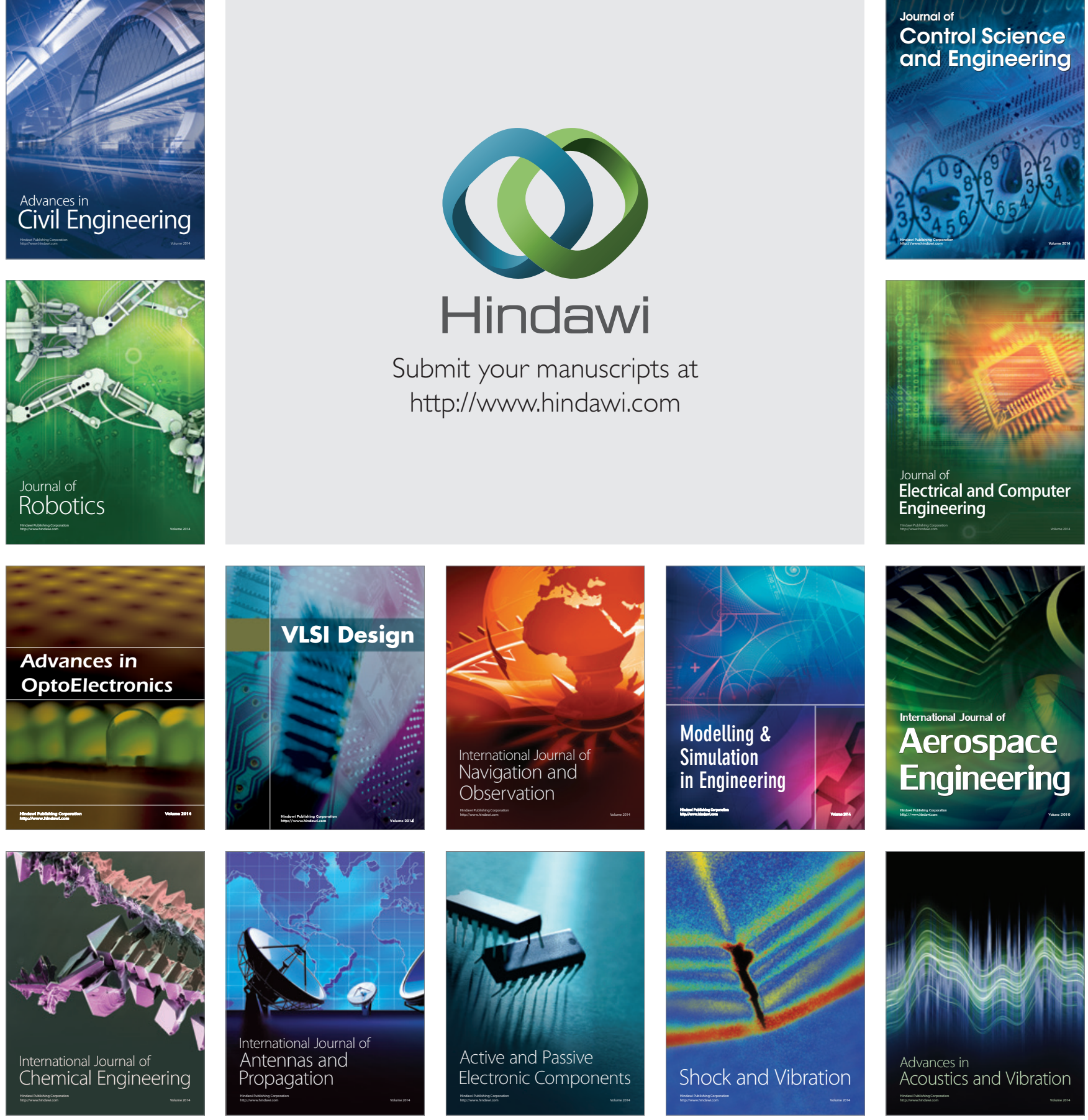